\title{
No difference at two years between all inside transtibial technique and traditional transtibial technique in anterior cruciate ligament reconstruction
}

\author{
Piero Volpi \\ Corrado Bait \\ Matteo Cervellin \\ Matteo Denti \\ Emanuele Prospero \\ Emanuela Morenghi \\ Alessandro Quaglia
}

Knee Orthopaedic and Sports Traumatology Unit, Humanitas Research Hospital, Rozzano, Italy

Corresponding author:

Piero Volpi

Knee Orthopaedic and Sports Traumatology Unit, Humanitas Research Hospital

20089 Rozzano, Italy

E-mail: piero.volpi@humanitas.it

\section{Summary}

Background: one of the most recent technique is the "all inside" anterior cruciate ligament (ACL) reconstruction. One of the main characteristic of this procedure is the sparing of the tibial cortex. Furthermore, the all-inside technique requires only one tendon harvested.

Purpose: the present study describes two year clinical outcomes of the all-inside method for ACL reconstruction, and compares them with clinical results of a group of patients treated with the traditional transtibial single-bundle $A C L$ reconstruction technique using the semitendinosus and gracilis tendons (ST-G).

Study design: pilot study, using historical controls. Methods: ACL reconstruction was performed on two groups of 20 patients each. The patients in one group underwent the all-inside transtibial technique with ST tendon alone. The second group underwent $A C L$ reconstruction with the traditional transtibial single-bundle procedure using quadrupled ST-G tendons. Follow up at 24 months was undertaken using the IKDC, VAS pain score, Lysholm and Tegner scales.

Results: the VAS pain score for the traditional ACL group was $84.6 \pm 12.6$; whereas the score for the all-inside group was $81.6 \pm 13.1$, with no statistically significant differences between the two groups. In the traditional $A C L$ reconstruction group the Lysholm scale gave a "good results" for 7 patient $(35 \%)$ and "excellent results" for 13 patients (65\%) and the all-inside group gave "sufficient results" for 4 patients (20\%), "good results" for 7 patients (35\%) and "excellent results" for 9 patients (45\%) (n.s.). The median of Tegner score was $6.5(2-10)$ for the standard method group and 6 (1-9) for the all-inside group (n.s). The IKDC evaluated $50 \%$ of patients from the standard technique group as class $A$, and $45 \%$ as class $B$ and $5 \%$ as class C. As regards patients of the all inside technique $55 \%$ were class A, $40 \%$ class B and, here too, just $5 \%$ scored as class C. No patients were classed as group $D$ in each group.

Conclusions: this study suggests that, in respect to return to sports and adequate articular function, there are no differences between the all-inside transtibial $A C L$ reconstruction technique and the traditional transtibial $A C L$ reconstruction using ST-G. The role of all-inside transtibial ACL reconstruction remains dubious.

Level of evidence: III or Level C according with Oxford Center of EBM.

KEY WORDS: anterior cruciate ligament reconstruction, all-inside technique, clinical outcome.

\section{Introduction}

Reconstruction of the anterior cruciate ligament ( $A C L)$ has undergone numerous innovations ${ }^{1}$. The procedure evolved from ACL reconstructions through arthrotomy to minimally invasive arthroscopic reconstructions. The choice of fixation methods moved from the use of interference metallic screws to reabsorbable screws, and finally to reabsorbable pins and suspension systems ${ }^{2,3}$, with no evidence of superior clinical results of one method of fixation over another. At the same time, some authors concentrated their efforts on restoring the anatomy, achieving optimal tunnel position ${ }^{4}$, promoting the use of double bundle techniques ${ }^{5-8}$, again with no clear evidence of superior clinical results of double over single bundle techniques.

A recent technique is the "all inside" ACL reconstruction $^{9}$, which spares the tibial cortex. Furthermore, the all-inside technique requires only one tendon to be harvested. This tendon can be used double, triple or even quadrupled because a shorter length is required than for traditional methods ${ }^{10}$.

When performed with allograft tissue, this technique requires only arthroscopic portals and no skin incisions are necessary. 
The "all inside" ACL reconstruction has been promoted by some authors as "no incision technique", purporting that the patients "may experience greater satisfaction as a result of improved cosmesis", 11 . The low invasiveness of the technique gives the added advantage of being able to associate it with other surgical procedures such as high tibial osteotomy or combined anterior and posterior cruciate ligament (ACL-PCL) reconstructions. These considerations lead the authors to include the all-inside ACL reconstruction technique among the options available to the knee surgeon especially when other procedure were associated, albeit technically demanding and requiring a steep learning curve ${ }^{12-14}$. We decided to perform a pilot study to compare the two year clinical outcomes of the all-inside transtibial method for $A C L$ reconstruction with the traditional transtibial single-bundle ACL reconstruction technique using the semitendinosus and gracilis tendons (ST-G).

\section{Materials and methods}

\section{Patients}

This pilot study was carried out on 20 patients between 2007 and 2008.

We included in our study male and female between 18 and 45 years old with clinical and radiological (MRI) ACL injury.

Exclusion criteria were revision surgery, inflammatory disease or other articular disease.

After undertaking the procedure on 10 patients (not included in this study), 20 patients were consecutively treated with $A C L$ reconstruction using the all-inside transtibial technique with ST autograft alone by the same fellowship trained surgeon (PV).

Thereafter, another 20 patients were consecutively treated with primary reconstruction of the anterior cruciate ligament of the knee with the traditional transtibial single-bundle procedure using ST-G autograft by the same surgeon.

Follow up after 24 months was made using the IKDC form $^{15}$, VAS pain score, Lysholm score ${ }^{16-18}$ (8 different items for a maximum score of 100). We considered a Lysholm score, between 84-94 as a good result, and $\geq 95$ as an excellent result.

Regarding IKDC form, we considered (in accordance with literature) class A as "normal" function and class $B$ as "nearly normal" function.

In addition, we recorded Tegner score ${ }^{16,17,19}$ to assess athletic and working activities with a score $\geq 5$ as good results. All evaluations were made at the 24 month follow-up by a blinded observer.

\section{Surgical technique}

All reconstructions were carried out by the same surgeon. All patients underwent routine arthroscopy.

Patients in the all inside group underwent the following procedure: after arthroscopic assessment, the semitendinosus tendon alone was harvested through an anteromedial oblique cutaneous incision, from proximal to distal over the pes anserinus and it was measured and prepared to a maximum length of 75 $\mathrm{mm}$. The diameter of the graft varied depending on whether the tendon was double or triple, but usually ranged between 7 and $8 \mathrm{~mm}$.

The notch was prepared and, where necessary, a notch-plasty was performed; the tibial guide (Arthrex Inc Naples, USA) was positioned at a $50^{\circ}$ angle, keeping it medial in respect to the major tibial axis. A cutter which was able to drill in two directions (dualcutter) was fixed on the tip of the guide pin. Placement of the dualcutter in the tibial area was accurately assessed, a graduated $3 \mathrm{~mm}$ diameter transtibial retrodrill pin was advanced through the tibial tunnel and hooked onto the dualcutter.

Keeping a firm hold of the guide, the surgeon drilled the tibia of the pre-established diameter, to match the graft length of $35 \mathrm{~mm}$ (Fig. 1). The metallic guide was removed and, using the same retrodrill pin as a guide and the same dualretrocutter hooked onto the retrodrill pin, the femoral half tunnel was drilled to $35 \mathrm{~mm}$ (Fig. 2). After unhooking the metallic wire, the dualcutter was retrieved from the joint with arthroscopic forceps.

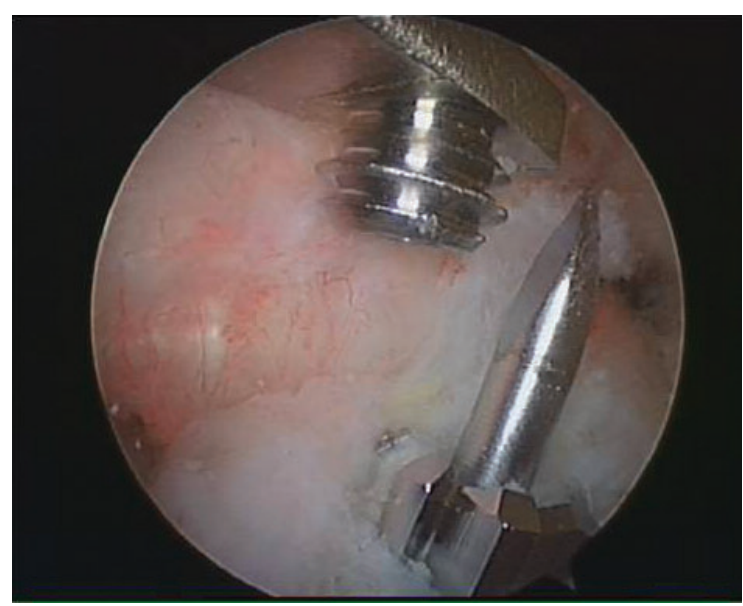

Figure 1. Drilling of the tibial tunnel with a retro cutter.

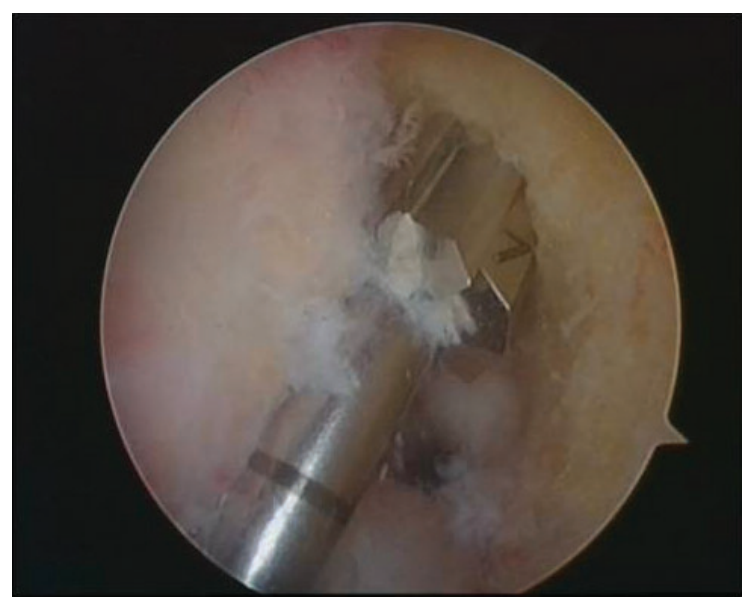

Figure 2. Transtibial drilling of femoral tunnel. 
No difference at two years between all inside transtibial technique and traditional transtibial technique in anterior cruciate ligament reconstruction

Graft length should ideally be $75 \mathrm{~mm}$, or, at least, 1.5 $\mathrm{cm}$ shorter than the sum of the two half tunnels and the joint to enable easy positioning of the femoral suspension fixation system and adequate tension.

The grafts were advanced into the joint via the anteromedial portal. Firstly, the graft was passed into the tibial half tunnel, and then the proximal part of the graft, containing the pre-prepared suspension fixation device, was introduced until it entered the femoral half tunnel.

Once the cortical femoral fixation device was positioned and tensioned distally, under arthroscopic control, the graft was fixed to the tibia with a metallic cortical suture button (Figs. 3, 4).

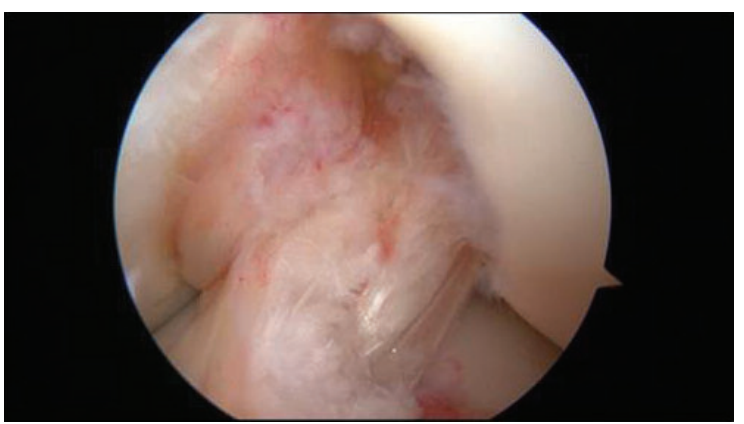

Figure 3. Arthroscopic final view.

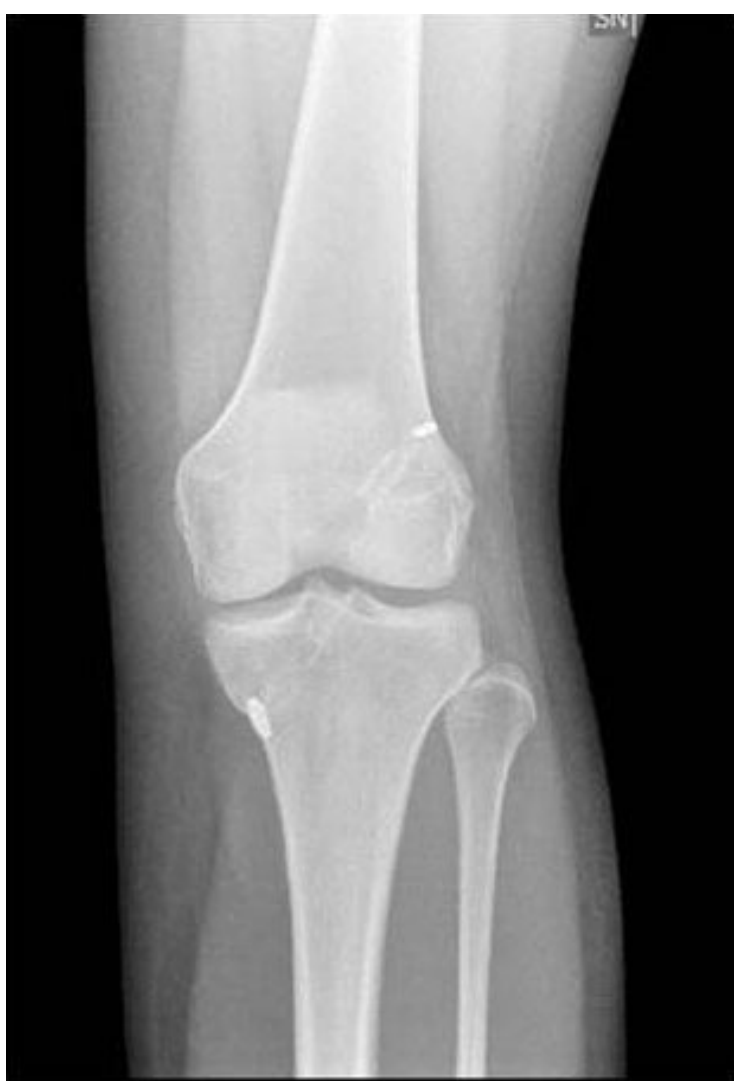

Figure 4. Radiographic A-P final view.
In accordance with the will to describe clinical outcomes of patients treated with traditional arthroscopic transtibial ACL single-bundle reconstruction using ST and $\mathrm{G}$ grafting, we evaluated another 20 patients.

On these patients, arthroscopic assessment was made. A longitudinal medial skin incision was made over the pes anserinus for ST and G tendon harvest for use as grafting material. The tibial tunnel and half femoral tunnel were then prepared using transtibial method after notch plasty when occurred. Once in position, the graft was fixed either with reabsorbable cross-pins in both the femur and the tibia, or by the suspension method at the level of the femur and with reabsorbable interference screws at the tibial level.

\section{Statistical analysis}

Data were expressed as number and percentage, or mean and standard deviation or median and range, where appropriate. Differences between groups were explored with t test, or Mann-Whitney test, or chi square test with Fisher correction if necessary, where appropriated. A p level of 0.05 was considered significative.

In accordance with international standards and as required by this journal, author declare that the study meets the ethical standards of this journal ${ }^{20}$.

\section{Results}

Twenty patients (12 men) underwent all inside transtibial technique. Mean age of patients was 38.4 \pm 10.8 years. No patients were lost to follow up.

Evaluation with VAS pain score for all-inside patients was $81.6 \mathrm{~mm} \pm 13.1$. Lysholm evaluation gave "sufficient results" for 4 patients (20\%), "good results" for 7 patients (35\%) and "excellent results" for 9 patients (45\%). Median Tegner score was 6 (1-9).

Using the IKDC form, 11 patients $(55 \%)$ were class $A$ ("normal" function), 8 patients $(40 \%)$ class B ("almost normal" function), and 1 patient (5\%) scored as class C ("abnormal" function). No patients were classed as group D ("severely abnormal" function).

Another group of 20 patients were treated with transtibial single bundle technique, whose 13 (65\%) men. Mean age of patients was $32.6 \pm 9.3$ years. No patients were lost to follow up.

Evaluation with the VAS pain score for the traditional ACL group, gave a score of $84.6 \mathrm{~mm} \pm 12.6$. The Lysholm scale gave a "good results" for 7 patient (35\%) and "excellent results" for 13 patients (65\%). The median Tegner score was 6.5 (2-10) for the standard method patients. Using the IKDC form, 10 patients $(50 \%)$ were classified as class A, 9 patients $(45 \%)$ as class $B$, and 1 patient $(5 \%)$ as class C. No patients were classified as class D.

No statistical differences for age, Lysholm, Tegner, VAS scale and IKDC results were found between all inside technique group and transtibial single bundle technique group (Tab. 1). 
Table 1. Summary results.

\begin{tabular}{llll}
\hline & Traditional ACL technique & All Inside technique & p value \\
\hline Age & $32.6 \pm 9.3$ & $38.4 \pm 10.8$ & 0.08 \\
\hline VAS & $84.6 \pm 12.7$ & $81.7 \pm 13.1$ & 0.47 \\
\hline Tegner & $6.4 \pm 2.3$ & $5.2 \pm 2.3$ & 0.11 \\
\hline Lysholm & $94.9 \pm 5.1$ & $90.9 \pm 8.8$ & 0.09 \\
\hline IKDC & $10(50 \%)$ & $11(55 \%)$ & 0.95 \\
A & $9(45 \%)$ & $8(40 \%)$ & \\
B & $1(5 \%)$ & $1(5 \%)$ & \\
C & & & \\
\hline
\end{tabular}

\section{Discussion}

For the variables examined in the present study, the allinside transtibial $A C L$ reconstruction technique is a good option for the knee surgeon in ACL reconstruction, because it gives overlapping result respect traditional transtibial single bundle technique. The increased difficulty of the procedure due to the high learning curve and possible technical errors make it advisable, in our opinion, that it be used only by expert.

Furthermore, one of the advantages of the all-inside technique is that it might be easily combined with surgeries such as high tibial osteotomy because of the sparing of tibial cortex or combined with ACL-PCL reconstructions. Moreover tibial drilling in all inside technique would appear to reduce the "blow-out fracture" phenomenon at the proximal tibia level21.

This, together with the fact that only the tibial half tunnel has been drilled, probably a smaller quantity of synovial fluid might invade the tibial socket. Whether this exerts a beneficial effect remains to be ascertained.

Respecting the tibial cortex reduces any eventual periosteal irritation, and probably leads to quicker recovery and greater patient satisfaction, but we acknowledge that we did not have data about this particular aspect. Moreover, the harvest of just one tendon avoids sacrificing a hamstring tendon. This aspect of reduced donor-site morbidity is even more important for young, athletic patients who need greater knee flexor integrity. When both hamstrings are removed, not only there can be greater postoperative donor site morbidity, but also strength deficit of the flexors. We acknowledge however that there are no level I studied to substantiate this.

Isokinetic assessments found flexor strength and internal rotation deficit equal to $5-10 \%$ compared with the contralateral limb (peaks under stress) ${ }^{22,23}$ : the actual clinical relevance of this deficit during normal daily activity or during sports is dubious.

Regards the use of a suture-button for tibial fixation, the authors proposed to not use interference screws, so as to take full advantage of the bone-tendon interface and hence profit from an improved osteointegration process. Indeed, a possible limitation of the use of hamstrings in $A C L$ reconstruction is that they needs a longer osteointegraton as well as more process than patellar tendon bone ${ }^{24,25}$.
The cost of single use devices (retrodrill pin, retrodualcutter, FiberWire) in addition to the common fixation systems is a deterrent in the routine use of this method, as is the longer time to undertake the procedure.

This pilot study has some limitations. First of all, it is not a randomized controlled trial. However, the present results could be used to make a more precise power calculation for further studies.

Another limit of this study is the presence of variability of fixation in the transtibial group. The relatively short follow-up makes it necessary to monitor the patient for a longer period to have more detailed data.

\section{Conclusion}

The results of this study seem suggest that, in respect to a return to sports activity, the all-inside transtibial $A C L$ reconstruction technique does not produce superior results to the traditional transtibial single bundle technique. This technique could be combined with surgeries such as osteotomy or ACLPCL reconstructions.

None of authors have conflict of interest in this matter.

\section{References}

1. Longo UG, Buchmann S, Franceschetti E, Maffulli N, Denaro V. A systematic review of single-bundle versus double-bundle anterior cruciate ligament reconstruction. Br Med Bull. 2012;103(1):147-168.

2. Volpi P, Marinoni L, Bait C, Galli M, de Girolamo L. Tibial fixation in anterior cruciate ligament reconstruction with bonepatellar tendon-bone and semitendinosus gracilis autografts: a comparison between bioabsorbable screws and bioabsorbable cross-pin fixation. Am J Sports Med 2009;37(4):808-812.

3. Capuano L, Hardy P, Longo UG, Denaro V, Maffulli N. No difference in clinical results between femoral transfixation and bio-interference screw fixation in hamstring tendon ACL reconstruction. A preliminary study. Knee. 2008;15:174-179.

4. Gougoulias N, Khanna A, Griffiths D, Maffulli N. ACL reconstruction: Can the transtibial technique achieve optimal tunnel positioning? A radiographic study. Knee. 2008;15(6):486490.

5. Colombet P, Robinson J, Christel P, et al. Morphology of anterior cruciate ligament attachments for anatomic reconstruction: a cadaveric dissection and radiographic study. Arthroscopy. 2006;22(9):984-992. 
No difference at two years between all inside transtibial technique and traditional transtibial technique in anterior cruciate ligament reconstruction

6. Crowrod C, Nylan J, Landes S, et al. Anatomic double bundle ACL reconstruction: a litterature review. Knee Surg Sports Traumatol Arthtrosc. 2007;15:946-964.

7. Meredick RB, Vance KS, Appleby D, Lubowitz JH. Outcome of single bundle versus double bundle reconstruction of the anterior cruciate ligament. Am J Sports Med. 2008;36(7):1414-1421.

8. Yagi M, Kuroda R, Nagamune K, Yoshiya S, Kurosaka M Double-bundle ACL reconstruction can improve rotational stability. Clin Orthop Relat Res 2007;454:100-107.

9. Lubowitz JH. No tunnel anterior cruciate ligament recontruction: The transtibial all-inside technique. Arthtroscopy. 2006;22:900e1-900e11.

10. Charalambous CP, Kwaees TA. Anatomical considerations in hamstring tendon harvesting for anterior cruciate ligament reconstruction. Muscles Ligaments Tendons J. 2013;2(4):253257.

11. Morgan CD. The all-inside ACL recontruction In: Operative technique Manual. Naples FLA: Arthrex Inc:1995.

12. Lubowitz JH. All-inside ACL: Reconstruction controversies. Sports Med Arthrosc Rev. 2010;18(1):20-26.

13. Morgan CD, Stein Da, Leitman EH, Kalman VH. Anatomic tibial graft fixation using a a retrograde bio-interference screw for endoscopic anterior cruciate ligament reconstruction. Arthroscopy. 2002;18:E38.

14. Stahelin A, Weiler A. All-inside anterior cruciate ligamnet reconstruction using a semitendinosus tendon and soft threaded biodegradable interference screw fixation. Arthroscopy. 1997;13:773-779.

15. Irganag JJ, Ho H, Harner CD, Fu Fh. Use of the International Knee Documentation Committee guidelines to assess outcome following anterior cruciate ligament reconstruction. Knee Surg Sports Traumatol Arthtrosc. 1998;6:9107-9114.

16. Lysholm and Tegner. Knee injury rating scales. Acta Orthopaedica. 2007;78(4):445-453.
17. Tegner $Y$, Lysholm J. Rating systems in the evaluation of knee ligament injuries. Clin Orthop. 1985;(198):43-49.

18. Lysholm J, Gillquist J. Evaluation of knee ligament surgery results with special emphasis on use of a scoring scale. Am J Sports Med. 1982;3(10):150-154.

19. Briggs KK, Lysholm J, Tegner Y, Rodkey WG, Kocher MS, Steadman JR. The reliability, validity, and responsiveness of the Lysholm score and Tegner activity scale for anterior cruciate ligament injuries of the knee: 25 years later. Am J Sports Med. 2009;37(5):890-897.

20. Padulo J, Oliva F, Frizziero A, Maffulli N. Muscle, Ligaments and Tendons Journal - Basic principles and recommendations in clinical and field science. Muscles Ligaments Tendons J.2013; 4:250-252.

21. McAdams T, Biswal S, Stevens $\mathrm{K}$ et al.Tibial aperture bone disruption after retrograde versus antegrade tibial tunnel drillig. Knee Sprts Traumatol Arthrosc.2008;16:818-822.

22. Kramer J, Nusca D, Fowler P, et al. Knee flexor and extensor strenght during concentric and eccentric muscle actions after anterior cruciate ligament recostruction using the semitendinosus tendon and ligament augmentation device. Am J Sports Med. 1993;21:285-291.

23. Segawa H, Omori G, Koga Y, Kameo T, lida S, Tanaka M. Rotational muscle strength of the limb after anterior cruciate ligament reconstruction using semitendinosus and gracilis tendon. Arthroscopy. 2002;18(2):177-182.

24. Lovric V, Kanazawa T, Nakamura Y, Oliver RA, Yu Y, Walsh WR. Effects of Gaps Induced Into the ACL Tendon Graft on Tendon-Bone Healing in a Rodent ACL Reconstruction Model.Muscles Ligaments Tendons J. 2012;1(3):91-99.

25. Goradia VK, Rochat MC, Grana WA, et al. Tendon-to-tendon healling of a semitendinosus tendon autograft used for ACL recontruction in a sheep model. Am J Knee Surg. 2000;13:143151. 